\title{
Intravenous Leiomyomatosis of Gonadal Vein: A Case Report
}

\author{
Maher Maaita ${ }^{\mathrm{a}}$, Susan Thabet ${ }^{\mathrm{a}, \mathrm{d}}$, Ehab Al-Rayyan ${ }^{\mathrm{a}}$, Abdullah Al-Omari ${ }^{\mathrm{b}}$, \\ William Haddadin ${ }^{\mathrm{c}}$
}

\begin{abstract}
Intravenous leiomyomatosis (IVL) is defined as a rare benign smooth muscle tumor, which can grow within systemic veins usually iliac veins into inferior vena cava and the right side of the heart; involvement of the gonadal veins is less commonly reported. We present a case with IVL and provide a review of the clinical presentation, pathology and post-operative issues. Operative management aims to completely resect all tumors in the safest manner for the patient.
\end{abstract}

Keywords: IVL; Fibroid; Uterine leiomyoma; Smooth muscle tumor; Ovarian vein

\section{Introduction}

Leiomyoma is the most common neoplasm in the female genital tract [1]. Intravenous leiomyomatosis (IVL) is a rare variant that holds benign histopathological features. When diagnosed late, the consequences could be life-threatening. It can grow into pelvic veins, inferior vena cava (IVC) and sometimes extends into the chambers of the heart and pulmonary vasculature. Due to its unusual nature, reports are usually limited to isolated cases or small series.

\section{Case Report}

A 42-year-old female, multiparous, previously healthy, presented with a history of prolonged heavy periods over the past 2 years. Pelvic ultrasound showed a complex left adnexal mass with a large uterine fibroid. Hysteroscopy and curettage revealed no malignant changes. Blood and biochemical markers were all normal.

Manuscript submitted September 24, 2018, accepted October 4, 2018

${ }^{a}$ Department of OBS/GYN, King Hussein Medical Center, Amman, Jordan ${ }^{b}$ Department of Radiology, King Hussein Medical Center, Amman, Jordan ${ }^{\mathrm{c}}$ Department of Histopathology, King Hussein Medical Center, Amman, Jordan

${ }^{\mathrm{d} C}$ Corresponding Author: Susan Thabet, Department of OBS/GYN, King Hussein Medical Center, Amman, Jordan. Email: Theflower123@yahoo.com

doi: https://doi.org/10.14740/jmc3174
Patient was counseled regarding options of management and opted for surgery. She was very keen to keep her right ovary.

During surgery, the uterus was noted to be slightly enlarged with asymmetrical outer surface. The upper fundal area and left parametrium showed a bulging mass extending into the broad ligament measuring $(6 \times 3.5 \mathrm{~cm})$. Both ovaries and fallopian tubes were normal. A total abdominal hysterectomy with left salpingo-oophorectomy was carried out as the mass was extending into the left infundibulopelvic and broad ligaments. The diagnosis of intravascular leiomyomatosis was not suspected before surgery.

On histopathological examination of the resected structures, the uterus and cervix measured $11 \times 8 \times 5 \mathrm{~cm}$. The cut surface of the uterus showed a large fungating submucosal fibroid projecting into the endometrial cavity measuring $8 \times 3$ $\times 2 \mathrm{~cm}$; multiple foci of adenomyosis and multiple nodular tumoral growths involving the outer lateral wall of the uterus with extension to the left broad ligament adjacent to the attached left ovary were found. The left parametrium showed dilated vascular spaces containing an intravascular fleshy tumor similar to that in the broad ligament which is consistent with intravascular leiomyomatosis (Fig. 1).

One-week post-surgery, the patient developed fever as a result of pelvic abscess formation that was drained through a small opening in the vaginal vault; subsequently, she improved. She underwent further imaging with CT angiogram, which revealed a tumoral thrombus in the left ovarian vein with normal left renal vein and IVC (Fig. 2), and no extension of the tumor to the cardiac chambers.

Six months after the last scan, another $\mathrm{CT}$ angiogram was

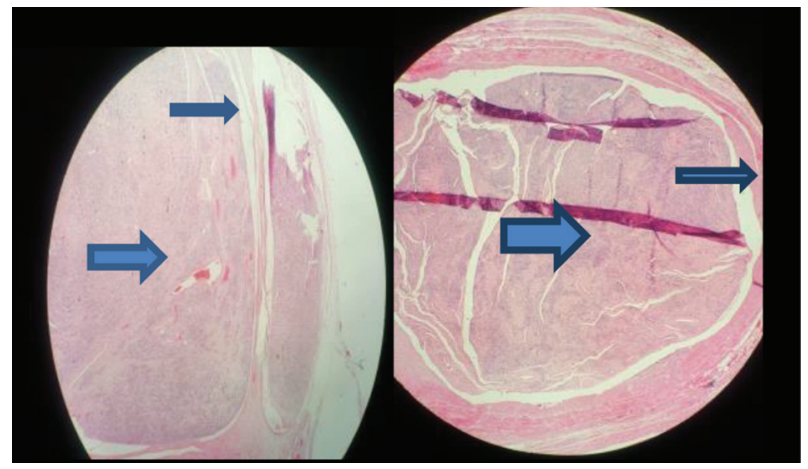

Figure 1. Histological findings revealing intravenous leiomyomatosis (large arrow) inside the vessel wall (small arrow). 


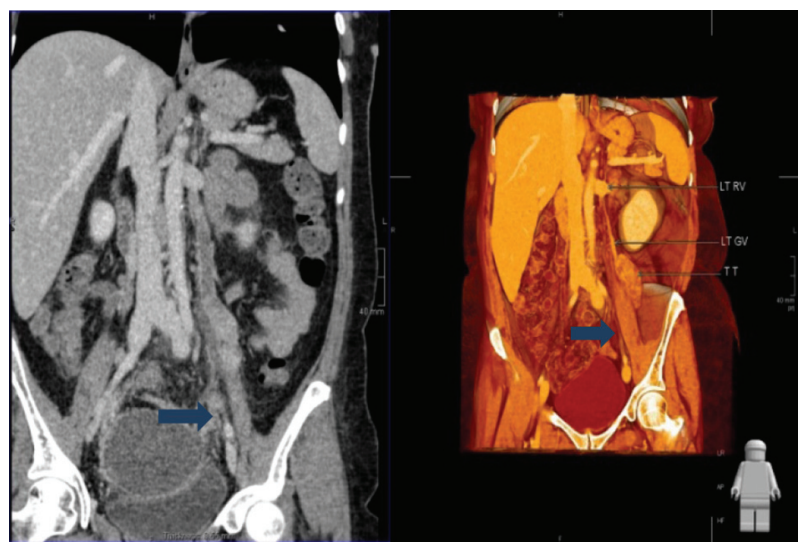

Figure 2. Enhanced CT revealed presence of tumor in left ovarian vein following first surgery.

done to assess the progress of the recurring tumor. A soft tissue attenuation enhancing mass measuring $12 \times 7 \times 7 \mathrm{~cm}$ anterior to the left psoas muscle was found (Fig. 3). The left ovarian vein above the tumor was normal with no evidence of tumoral extension into its superior aspect, with normal left renal vein and IVC.

The patient remained asymptomatic during this period and was re-operated again to resect the recurring tumor. A welldefined retroperitoneal mass measuring $11 \times 8 \times 5 \mathrm{~cm}$ was excised presenting a benign leiomyoma (Fig. 4), part of which appeared to be arising from intravascular location with no evidence of malignancy.

The patient made good recovery and was discharged home on day 3 post-surgery. She was reviewed in OPD 4 weeks after surgery and was in good general condition. She was given an appointment to be reviewed in 6 months.

\section{Discussion}

In 1896, Birch-Hirschfeld was the first to report a case that is related to the clinical features of IVL. IVL is defined as a benign smooth muscle cell tumor that grows along the uterus

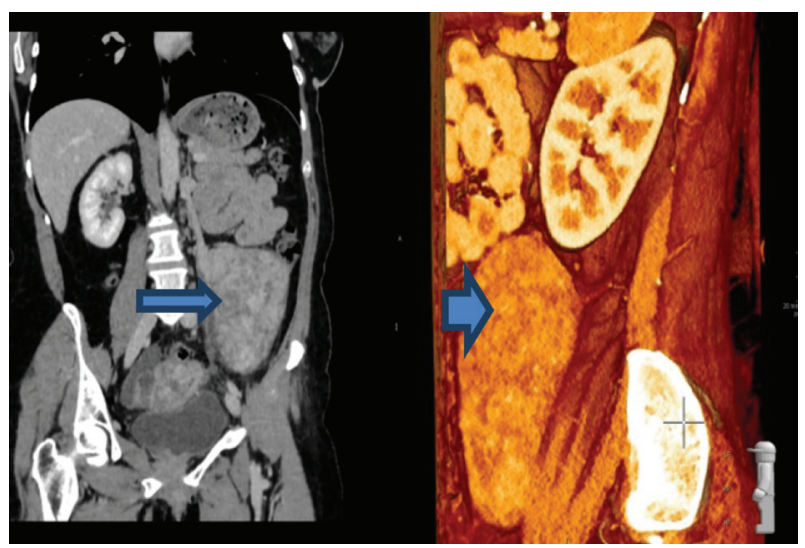

Figure 3. A mass measuring $12 \times 7 \times 7 \mathrm{~cm}$ anterior to the left psoas muscle.

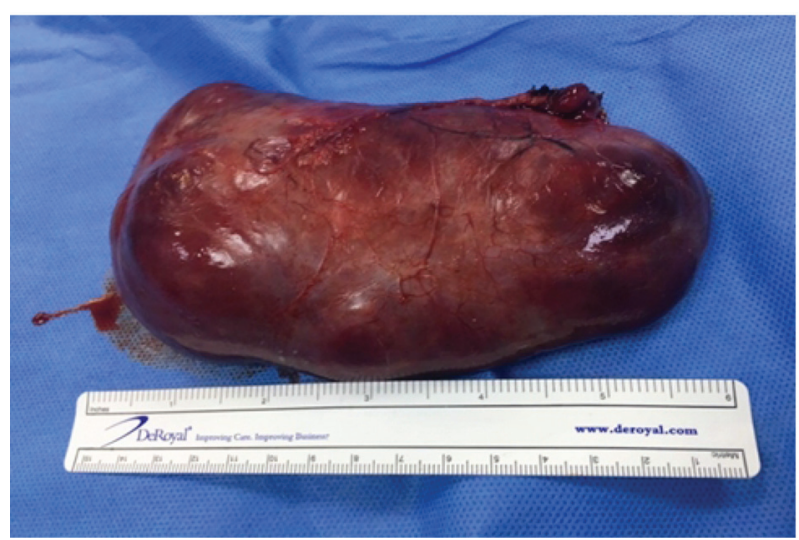

Figure 4. Intravascular leiomyomatosis removed in the second surgery.

or extra uterine venous system, or both $[2,3]$. In 1907, the first case of IVL with intracardiac extension was reported by Durck [4]. The tumors, which were described as nodular or spiral, involved the pelvic veins, IVC, or even extended to the right heart chambers and pulmonary vessels [5-7].

Regarding the etiology of IVL, two main hypotheses have been proposed. The first one was suggested by Knauer, who proposed that the neoplasm originates from estrogen-induced smooth muscle cell proliferation in the walls of uterine veins [8], while the second hypothesis declared that the primary tumor originated from uterine leiomyoma that projected into the adjacent venous system [9]. Fukuyama et al [10] proposed that the neoplasm does not break through the vessel wall, but rather stretches along the vascular wall to extend along the lumen of the blood vessel while covered in endothelium.

Invasion of pelvic veins and IVC by the tumor may result in wide spectrum of symptoms, ranging from pelvic pain and abnormal uterine bleeding to shortness of breath, edema of the lower limbs or even sudden death. The clinical manifestations mainly depend on the degree of intravascular obstruction caused by the tumor. However, most cases were diagnosed intraoperatively.

In most of case studies, the initial pathway of extension for IVL was unilateral through the lumen of uterine and iliac veins into IVC. However, less commonly the ovarian veins can serve as an alternative route to the IVC [11]. In the presented case, the tumor was expected to extend from the left ovarian vein into the left renal vein, and then IVC.

Imaging studies are essential for the diagnosis of IVL. Ultrasound and echocardiography are useful for evaluating the tumor size, nature and extension to the cardiac chambers. CT and MRI are the most useful imaging modalities for identifying the continuity of intraluminal tumors.

The differential diagnosis of IVL should include intravenous thrombi leiomyosarcoma originating from the wall of IVC, right atrial myxoma and malignant thrombosis with carcinoma [12], as their CT and MRI features are similar to IVL. However, thrombi within systemic veins show no enhancement following contrast agent administration due to their lack of vascular supply. It could be challenging to distinguish between IVL and leiomyosarcoma of IVC, especially in the early stages of the disease, nevertheless, when leiomyosarcoma 
presents with obvious infiltration and invasion of surrounding structures diagnosis becomes more simple [13]. Right atrial myxoma typically involves the heart and does not extend into IVC. Malignant thrombi originate from primary site of cancer and generally are considerably smaller than the IVL. Furthermore, malignant thrombosis is not associated with the presence of uterine fibroid or history of uterine fibroid surgery [14].

Complete surgical resection of intravenous leiomyomatosis is the key to successful treatment [15], and the final diagnosis depends on histopathological examination. Estrogen and progesterone receptor expression in the tumor cells is considered as a good prognostic factor; therefore, treatment with anti-estrogen may be effective. According to a case report by Tresukosol et al [16], leuprolide acetate was given preoperatively resulting in a significant reduction in the tumor size. So anti-estrogen therapy can be justified in patients with incompletely resected tumor or residual tumor cells, keeping in mind that these patients will need a careful follow-up for several years. Evan et al [17] studied four cases with recurrent pelvic IVL and a functioning ovarian tissue was found at the time of recurrence in three of them; hence, a bilateral oophorectomy was suggested to prevent recurrence of the tumor.

When complete resection is thought to have been achieved, recurrence of IVL seems to be a rare event. Ahmed et al [18] reported a recurrence rate of about $30 \%$; therefore, follow-up is essential. The most common imaging modality for follow-up in reported papers is CT scan. MRI, ultrasound and echocardiography are also reported in use. Where complete resection is achieved, repeated imaging on 6-monthly to -yearly basis, and to investigate symptoms is appropriate. In cases with incomplete resection, especially when intravascular tumor remains, radiological follow-up should be more frequent.

In this case, diagnosis of IVL was not suspected preoperatively and was confirmed only by histopathological examination postoperatively. Therefore, the diagnosis of IVL should be considered during surgery where uterine fibroids seem to extend beyond the uterus into para-uterine tissues.

The patient did well after two surgeries. She was counseled carefully about the rarity of the disease, the need for close and long-term follow-up, and the risk of right oophorectomy if she developed any recurrence in the future. The patient was followed up every 6 months and she was asked to report any symptoms especially cardiac. Hormonal treatment was not discussed with the patient.

In conclusion, IVL is a rare variant of leiomyoma that better be considered upon presentation of a soft tissue mass in the systemic veins, with or without extension to the heart, in female patients, particularly those with a history of uterine myoma. The diagnosis can be easily missed preoperatively which makes the surgery more challenging and risky to the patient. Close and long-term follow-up is crucial.

\section{References}

1. Robboy SJ, Bentley RC, Butnor K, Anderson MC. Pathology and pathophysiology of uterine smooth-muscle tumors. Environ Health Perspect. 2000;108(Suppl 5):779-
784.

2. Norris HJ, Parmley T. Mesenchymal tumors of the uterus. $\mathrm{V}$. Intravenous leiomyomatosis. A clinical and pathologic study of 14 cases. Cancer. 1975;36(6):2164-2178.

3. Diakomanolis E, Elsheikh A, Sotiropoulou M, Voulgaris Z, Vlachos G, Loutradis D, Michalas S. Intravenous leiomyomatosis. Arch Gynecol Obstet. 2003;267(4):256257.

4. Harris LM, Karakousis CP. Intravenous leiomyomatosis with cardiac extension: tumor thrombectomy through an abdominal approach. J Vasc Surg. 2000;31(5):1046-1051.

5. Rajaii-Khorasani A, Kahrom M, Hashemzadeh M, Tayebi S, Ghazi M, Hamedanchi A. Pulmonary artery extension of uterine leiomyoma. J Card Surg. 2012;27(4):466-469.

6. Borland DS, Wotring JW. Intravenous Leiomyomatosis of the Uterus and Broad Ligament. Report of a Case. Am J Clin Pathol. 1964;42:182-188.

7. Lee S, Kim DK, Narm KS, Cho SH. Pulmonary artery embolization of intravenous leiomyomatosis extending into the right atrium. Korean J Thorac Cardiovasc Surg. 2011;44(3):243-246.

8. Knauer E. Beitragzuranatomie der uterusmyome. Beitr Geburtsh Gynaekol. 1903;1:695.

9. Sitzenfry A. Uebervenenmyome des uterus mitintravaskularemwachstum. Z Gerburtsh Gynaekol. 1911;68:1-25.

10. Fukuyama A, Yokoyama Y, Futagami M, Shigeto T, Wada R, Mizunuma H. A case of uterine leiomyoma with intravenous leiomyomatosis - histological investigation of the pathological condition. Pathol Oncol Res. 2011;17(1):171-174.

11. Lam PM, Lo KW, Yu MY, Wong WS, Lau JY, Arifi AA, Cheung TH. Intravenous leiomyomatosis: two cases with different routes of tumor extension. J Vasc Surg. 2004;39(2):465-469.

12. Bilyeu SP, Bilyeu JD, Parthasarathy R. Intravenous lipoleiomyomatosis. Clin Imaging. 2006;30(5):361-364.

13. McDonald DK, Kalva SP, Fan CM, Vasilyev A. Leiomyosarcoma of the uterus with intravascular tumor extension and pulmonary tumor embolism. Cardiovasc Intervent Radiol. 2007;30(1):140-142.

14. Osawa H, Hosaka S, Akashi O, Furukawa H, Egi K. A case of intravenous leiomyomatosis of uterine origin, extending through the inferior vena cava to right atrium. Gen Thorac Cardiovasc Surg. 2013;61(2):104-107.

15. Kocica MJ, Vranes MR, Kostic D, Kovacevic-Kostic N, Lackovic V, Bozic-Mihajlovic V, Velinovic MM, et al. Intravenous leiomyomatosis with extension to the heart: rare or underestimated? J Thorac Cardiovasc Surg. 2005;130(6):1724-1726.

16. Tresukosol D, Kudelka AP, Malpica A, Varma DG, Edwards CL, Kavanagh JJ. Leuprolide acetate and intravascular leiomyomatosis. Obstet Gynecol. 1995;86(4 Pt 2):688-692.

17. Evans AT, 3rd, Symmonds RE, Gaffey TA. Recurrent pelvic intravenous leiomyomatosis. Obstet Gynecol. $1981 ; 57(2): 260-264$.

18. Ahmed M, Zangos S, Bechstein WO, Vogl TJ. Intravenous leiomyomatosis. Eur Radiol. 2004;14(7):1316-1317. 